\title{
Independent confirmation of juvenile idiopathic arthritis genetic risk loci previously identified by immunochip array analysis
}

\author{
Rachel C Chiaroni-Clarke ${ }^{1,2}$, Jane E Munro ${ }^{3,4}$, Raul A Chavez ${ }^{1,2}$, Angela Pezic $^{5}$, Roger C Allen ${ }^{3,4}$, \\ Jonathan D Akikusa ${ }^{3,4}$, Susan E Piper ${ }^{6}$, Richard Saffery ${ }^{7,2}$, Anne-Louise Ponsonby ${ }^{5,2}$ and Justine A Ellis i, $^{1 *}$
}

\begin{abstract}
Background: Our understanding of the genetic factors underlying juvenile idiopathic arthritis (JIA) is growing, but remains incomplete. Recently, a number of novel genetic loci were reported to be associated with JIA at (or near) genome-wide significance in a large case-control discovery sample using the Immunochip genotyping array. However, independent replication of findings has yet to be performed. We therefore attempted to replicate these newly identified loci in the Australian CLARITY JIA case-control sample.

Findings: Genotyping was successfully performed on a total of 404 JIA cases (mean age 6.4 years, 68\% female) and 676 healthy child controls (mean age 7.1 years, 42\% female) across 19 SNPs previously associated with JIA. We replicated a significant association ( $p<0.05$, odds ratio $(O R)$ in a direction consistent with the previous report) for seven loci, six replicated for the first time - C5orf56-IRF1 (rs4705862), ERAP2-LNPEP (rs27290), PRR5L (rs4755450), RUNX1 (rs9979383), RUNX3 (rs4648881), and UBE2L3 (rs2266959).

Conclusions: We have carried out the first independent replication of association for six genes implicated in JIA susceptibility. Our data significantly strengthens the evidence that these loci harbor true disease associated variants. Thus, this study makes an important contribution to the growing body of international data that is revealing the genetic risk landscape of JIA.
\end{abstract}

Keywords: Juvenile idiopathic arthritis, Immunochip, Independent replication, Genetic association

\section{Findings}

Juvenile Idiopathic Arthritis (JIA) is the most common rheumatic disease in children [1], yet there are relatively few confirmed disease associated loci, and we are therefore limited in our understanding of the overall genetic architecture [2,3]. Only two genome-wide association studies have been published for JIA [4,5], limited by sample size and/or genomic coverage. In these studies, a discovery sample was used to identify potential risk loci, and then an independent replication sample was used to confirm the findings. In both studies, a number of loci were identified as associated with JIA in the discovery

\footnotetext{
*Correspondence: justine.ellis@mcri.edu.au

'Genes, Environment \& Complex Disease, Murdoch Childrens Research Institute, 50 Flemington Rd, Parkville, Victoria 3052, Australia

${ }^{2}$ Department of Paediatrics, The University of Melbourne, Parkville, Vic 3010, Australia

Full list of author information is available at the end of the article
}

sample that were unable to be replicated in the validation sample $[4,5]$. This highlights the importance of independent replication in determining true disease associated loci.

In 2013, Hinks et al. published the most successful genetic association study for JIA to date [6], using the Immunochip [7]; a genotyping array specifically designed to densely capture variation at immune-related genetic loci. The study further validated the well-established associations of HLA, PTPN22 and PTPN2 with JIA, in addition to confirming the involvement of other loci such as STAT4 with JIA, that were previously associated with the disease, but not at a genome-wide level of significance $\left(\mathrm{p}<5 \times 10^{-8}\right)[6]$. More notably, the same study identified a suite of novel JIA risk loci reaching either genome-wide significance, or a genome-wide 'suggestive' level of significance $\left(\mathrm{p}<1 \times 10^{-6}\right)$ [6]. However, in order to maximize statistical power for discovery, all 
available JIA cases formed the discovery sample, and no replication sample was available to confirm these novel associations. Therefore, we aimed to confirm the involvement of these new JIA risk loci in an independent sample of JIA cases and healthy child controls drawn from the CLARITY JIA case-control study in Melbourne Australia $[8,9]$.

We selected 22 single nucleotide polymorphisms (SNPs) from the JIA Immunochip association study [6], for potential replication. SNPs that are established susceptibility loci for JIA [4], or that have previously been associated in our sample [8] (either the SNP itself or a SNP in high linkage disequilibrium) were omitted. Two SNPs selected for inclusion subsequently failed assay design and were therefore also excluded. Reasons for exclusion are listed in Additional file 1: Table S1. Genotyping of the remaining 20 SNPs was attempted in a total of 408 JIA cases and 687 controls drawn from CLARITY, using the Sequenom MassARRAY system (assay design details available from the authors). Following exclusion of individuals and SNPs not meeting quality control thresholds $(<90 \%$ call rate for individuals or SNPs, and departure from Hardy Weinberg equilibrium $\mathrm{p}<0.001$ ), genotype data was successfully generated for 404 JIA cases (mean age 6.4 years, 68\% female) and 676 healthy child controls (mean age 7.1 years, $42 \%$ female) across 19 SNPs (listed in Table 1). One SNP, rs66718203 at PRM1-RM12, failed genotyping quality control and was excluded from further analysis. We regenotyped a random $10 \%$ selection of samples across all SNPs and achieved a genotype concordance rate of $>99 \%$. Allelic, genotypic, additive (Cochrane-Armitage test for trend), dominant and recessive association tests were performed using PLINK v1.07 [10]. We considered $\mathrm{p}<0.05$ for any test, along with an allelic odds ratio (OR) in a direction consistent with the previous report, as evidence of replication. As these SNPs had a priori evidence for association, we did not adjust the p values for multiple testing.

Table 1 shows the ORs and $p$ values for allelic association tests, as well as the 'best test' model and corresponding $\mathrm{p}$ value. These are shown alongside the reported ORs, $\mathrm{p}$ value, and model from the Immunochip study. Figure 1 shows a forest plot of the allelic odds ratios generated in the CLARITY sample compared to the Immunochip study ORs for all SNPs tested. We report replication of association for six genes (SNPs) in our sample - AFF3-LONRF2 (rs6740838), C5orf56-IRF1 (rs4705862), ERAP2-LNPEP (rs27290), PRR5L (rs4755450), RUNX1 (rs9979383) and UBE2L3 (rs2266959). A further seven genes (SNPs) showed

Table 1 CLARITY association replication results

\begin{tabular}{|c|c|c|c|c|c|c|c|c|c|}
\hline \multirow[t]{2}{*}{ Gene } & \multirow[t]{2}{*}{ SNP } & \multirow{2}{*}{$\begin{array}{l}\text { Minor } \\
\text { allele }\end{array}$} & \multirow{2}{*}{$\begin{array}{l}\text { Case } \\
\text { MAF/Control } \\
\text { MAF }\end{array}$} & \multirow[b]{2}{*}{$\mathbf{P}$} & \multirow{2}{*}{$\begin{array}{l}\text { Allelic } \\
\text { OR (95\% CI) }\end{array}$} & \multirow{2}{*}{$\begin{array}{l}\text { Best } \\
\mathrm{P}^{\mathrm{a}}\end{array}$} & \multicolumn{2}{|l|}{ Immunochip } & \multirow[t]{2}{*}{ Replication ${ }^{c}$} \\
\hline & & & & & & & $\mathrm{P}^{\mathrm{b}}$ & OR $(95 \% \mathrm{Cl})$ & \\
\hline $13 q 14$ & rs34132030 & $\mathrm{T}$ & $0.30 / 0.30$ & 0.82 & $1.02(0.85-1.24)$ & $0.68 \mathrm{D}$ & $1.77 \times 10^{-7} \mathrm{~A}$ & $1.18(1.11-1.26)$ & $\mathrm{N}$ \\
\hline AFF3-LONRF2 & rs6740838 & $\mathrm{T}$ & $0.44 / 0.41$ & 0.25 & $1.11(0.93-1.32)$ & $0.042 R$ & $8.83 \times 10^{-7} \mathrm{D}$ & $1.25(1.14-1.37)$ & Y \\
\hline ANKRD55 & rs71624119 & A & $0.20 / 0.23$ & 0.10 & $0.83(0.67-1.03)$ & $0.081 R$ & $4.40 \times 10^{-11} \mathrm{~A}$ & $0.78(0.73-0.84)$ & S \\
\hline ATP8B2-IL6R & rs11265608 & A & $0.08 / 0.07$ & 0.50 & $1.12(0.81-1.55)$ & $0.50 \mathrm{~T}$ & $2.75 \times 10^{-8} \mathrm{D}$ & $1.33(1.20-1.47)$ & N \\
\hline C5orf56-IRF1 & rs4705862 & $\mathrm{T}$ & $0.42 / 0.45$ & 0.11 & $0.87(0.73-1.04)$ & $0.033 \mathrm{D}$ & $1.02 \times 10^{-8} \mathrm{~A}$ & $0.84(0.79-0.89)$ & Y \\
\hline CCR1-CCR3 & rs79893749 & $\mathrm{T}$ & $0.09 / 0.11$ & 0.10 & $0.78(0.58-1.05)$ & $0.10 \mathrm{~A}$ & $1.88 \times 10^{-7} \mathrm{~A}$ & $0.78(0.72-0.86)$ & S \\
\hline ERAP2-LNPEP & rs27290 & G & $0.47 / 0.39$ & 0.00021 & $1.40(1.17-1.66)$ & $0.00021 \mathrm{~A}$ & $7.50 \times 10^{-9} \mathrm{D}$ & $1.32(1.20-1.45)$ & Y \\
\hline FAS & rs7069750 & G & $0.47 / 0.45$ & 0.19 & $1.12(0.94-1.34)$ & $0.19 \mathrm{~A}$ & $2.93 \times 10^{-8} \mathrm{~A}$ & $1.18(1.11-1.25)$ & S \\
\hline IL2-IL21 & rs1479924 & G & $0.27 / 0.28$ & 0.54 & $0.94(0.77-1.15)$ & $0.16 R$ & $6.24 \times 10^{-11} \mathrm{~A}$ & $0.79(0.74-0.85)$ & S \\
\hline IL2RB & rs2284033 & A & $0.40 / 0.43$ & 0.24 & $0.90(0.75-1.08)$ & $0.18 \mathrm{D}$ & $1.55 \times 10^{-8} \mathrm{~A}$ & $0.84(0.79-0.89)$ & S \\
\hline IL6 & rs7808122 & $\mathrm{T}$ & $0.41 / 0.40$ & 0.76 & $1.03(0.86-1.23)$ & $0.59 \mathrm{R}$ & $5.80 \times 10^{-8} \mathrm{~A}$ & $1.19(1.11-1.25)$ & N \\
\hline JAZF1 & rs10280937 & C & $0.15 / 0.14$ & 0.77 & $1.04(0.81-1.33)$ & $0.74 \mathrm{D}$ & $6.60 \times 10^{-7} \mathrm{~A}$ & $1.25(1.15-1.37)$ & N \\
\hline LTBR & rs2364480 & $C$ & $0.26 / 0.27$ & 0.65 & $0.96(0.78-1.16)$ & $0.49 R$ & $5.10 \times 10^{-8} \mathrm{~A}$ & $1.20(1.12-1.28)$ & N \\
\hline PRR5L & rs4755450 & A & $0.34 / 0.38$ & 0.043 & $0.83(0.69-0.99)$ & $0.042 \mathrm{D}$ & $3.35 \times 10^{-7} \mathrm{D}$ & $0.80(0.74-0.87)$ & Y \\
\hline RUNX1 & rs9979383 & C & $0.32 / 0.37$ & 0.013 & $0.79(0.66-0.95)$ & $0.013 \mathrm{~A}$ & $1.06 \times 10^{-8} \mathrm{D}$ & $0.78(0.72-0.85)$ & Y \\
\hline RUNX3 & rs4648881 & A & $0.48 / 0.50$ & 0.37 & $0.92(0.78-1.10)$ & $0.19 \mathrm{D}$ & $4.66 \times 10^{-7} \mathrm{~A}$ & $1.16(1.10-1.23)$ & $\mathrm{S}^{*}$ \\
\hline TYK2 & rs34536443 & C & $0.02 / 0.03$ & 0.10 & $0.63(0.36-1.10)$ & $0.10 \mathrm{~A}$ & $1.00 \times 10^{-10} \mathrm{~A}$ & $0.56(0.47-0.67)$ & S \\
\hline UBE2L3 & rs2266959 & $\mathrm{T}$ & $0.23 / 0.19$ & 0.040 & $1.25(1.01-1.55)$ & $0.036 \mathrm{~T}$ & $6.20 \times 10^{-9} \mathrm{D}$ & $1.24(1.15-1.33)$ & Y \\
\hline ZFP36L1 & rs12434551 & $\mathrm{T}$ & $0.46 / 0.45$ & 0.61 & $1.05(0.88-1.25)$ & $0.50 \mathrm{R}$ & $1.59 \times 10^{-8} \mathrm{D}$ & $0.77(0.71-0.85)$ & N \\
\hline
\end{tabular}

${ }^{a}$ Model providing most significant $\mathrm{P}$ value: $\mathrm{A}=$ allelic, $\mathrm{D}=$ dominant, $\mathrm{G}=$ genotypic, $\mathrm{R}=$ recessive, $\mathrm{T}=$ trend (Cochrane-Armitage Trend Test, also referred to as additive).

Immunochip model: $A$ = additive, $D=$ dominant.

'Evidence for replication $\mathrm{Y}=$ yes $\mathrm{N}=$ no $\mathrm{S}=$ suggestive ${ }^{*}=$ opposite direction of effect due to the minor allele being opposite between the two studies.

Bold = reached genome-wide significance in Immunochip paper. 


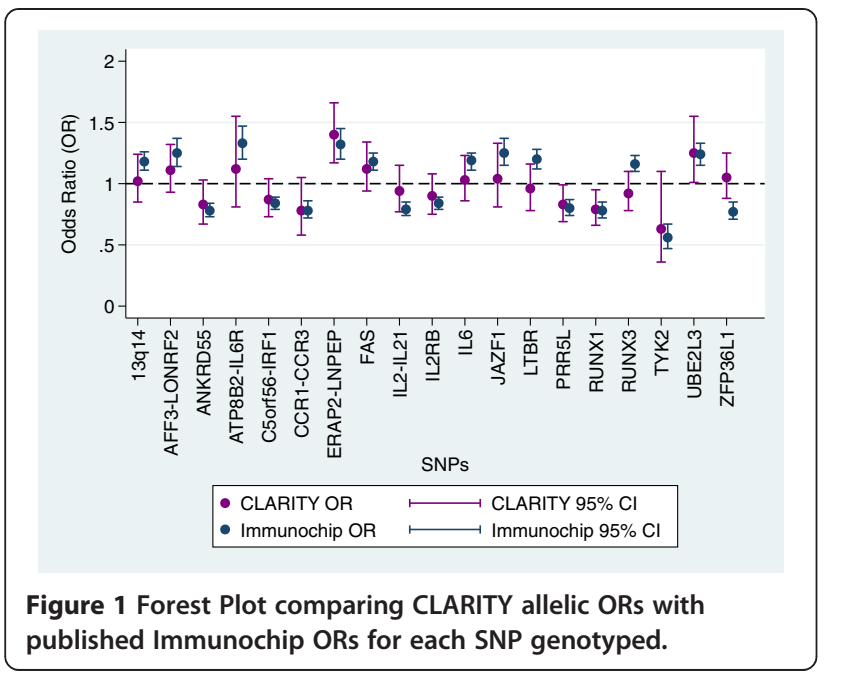

suggestive association (defined as an OR in a consistent direction and a $\mathrm{p}<0.2$ ) - ANKRD55 (rs71624119), CCR1CCR3 (rs79893749), FAS (rs7069750), IL2-IL21 (rs1479924), IL2RB (rs2284033), RUNX3 (rs4648881) and TYK2 (rs34536442).

We re-analysed the data after restricting the samples to those of European descent to ensure associations were not being masked by differences in ethnicity between the prior study and our own. Only those individuals who identified all four grandparents as European were included. Individuals with a non-European grandparent or any missing information were excluded. This restriction criterion reduced our sample size to 229 cases (mean age 6.2 years, $70 \%$ female) and 418 controls (mean age 7.2 years, $40 \%$ female). The results of this analysis are detailed in Additional file 1: Table S2. Overall, the results of this analysis did not considerably differ from the entire sample, with a few exceptions. Of note, RUNX3 (rs4648881), which had a suggestive association in the total sample, showed a significant association in the European-only sample. Furthermore, ZFP36L1 (rs12434551) showed significant association with JIA in the Europeanonly sample, suggesting it may be particularly sensitive to ethnicity. However, our reported SNP (T) differs to the previously reported SNP (A), and the association is in the opposite direction. As this SNP is an A/T transversion with frequencies of both alleles near $50 \%$ we cannot determine whether the direction of effect difference is due to allele reversal, and thus replicates the prior result, or the result of analysing different DNA strands between the two studies, in which case the risk locus is not replicated. Two genes that were significantly associated in the entire sample, PRR5L (rs4755450), and AFF3-LONRF2 (rs6740838), were no longer significant in the European-only analysis, and C5orf56-IRF1 (rs4705862) showed only suggestive significance. Given that the reduced sample size likely resulted in a reduction in statistical power, small movements in the ORs and $p$ values are not unexpected, and this result is unlikely to indicate failure to replicate.

We have replicated the association of AFF3-LONRF2 (rs6740838), and showed suggestive association of ANKRD55 (rs71624119) and IL2-IL21 (rs1479924), which all had evidence of association with JIA prior to the Immunochip study [11-13], although the samples used in those prior studies significantly overlapped with those used in the Immunochip study (i.e. the replication efforts were not independent). A recent independent study by Reinards et al. [14] also examined evidence for association of JIA with SNPs at ANKRD55 and AFF3 that are highly correlated $\left(\mathrm{r}^{2} \sim 0.9\right)$ with the Immunochip SNPs at these genes. The association with ANKRD55 was replicated, further supporting a role for this gene in JIA risk. The association with AFF3 was not replicated, although the direction of effect is consistent across studies.

Although our study has been able to confirm association of a number of Immunochip-identified genes with JIA risk, lack of replication in our study cannot be taken as evidence to refute association for a number of reasons. First, our sample size is much smaller than that used by the Immunochip study, and therefore has lower statistical power to detect association. This is somewhat offset by the more relaxed level of significance required for replication, compared to that required in the discovery study. Second, the Immunochip study was restricted to oligoarticular and polyarticular RF negative JIA. Here we have considered all JIA subtypes together. We chose not to perform subtype specific analyses, as the benefit of reduced heterogeneity would be offset by the reduction in statistical power associated with reduced sample size. The fact that the associations identified in this study were still detectable when all subtypes were considered together suggests common pathways to disease in all subtypes (potential 'pan-JIA' genes). Such genes are not unexpected, given the significant overlap amongst disease risk genes across far more clinically heterogeneous forms of autoimmune disease [15]. Despite the sample size and case heterogeneity limitations of our study, it is worth noting that a number of the associations that we have failed to replicate only carried a suggestive level of association in the original study, and therefore our lack of replication may well be indicating lack of true association.

This study has further confirmed association of JIA with seven genetic loci, six of which have now been replicated for the first time (C5orf56-IRF1 (rs4705862), ERAP2LNPEP (rs27290), PRR5L (rs4755450), RUNX1 (rs9979383), RUNX3 (rs4648881), and UBE2L3 (rs2266959)). For an additional six genes, we generated weaker indication of association, further supporting (but not yet confirming) involvement in JIA risk. Further analysis of these loci in other independent samples will assist in clarifying their role in disease susceptibility. Overall, our study makes 
an important contribution to the growing body of international data that is revealing the genetic risk landscape of this paediatric autoimmune disease.

\section{Additional file}

Additional file 1: Table S1. Significant/suggestive SNPs from the Immunochip study not included in this replication analysis. Table S2: CLARITY SNP replication results in European---restricted cohort, case $n=220$ control $n=418$.

\section{Competing interests}

The authors declare that they have no competing interests.

\section{Authors' contributions}

JE and RCC conceived, designed and led the current analysis. JE and JM led the CLARITY study from which the participants were drawn. ALP led control collection, and advised and assisted with study design and collection of epidemiological data. RCC performed the SNP genotyping. RC managed biospecimens. AP managed the study data. RA and JA assisted with case study design, and JM, RA, JA and SP assisted with case recruitment through their paediatric rheumatology clinics. RS contributed to study design and supervision. RCC and JE wrote the manuscript, and all authors participated in drafting the manuscript to the final version. All authors read and approved the final manuscript.

\section{Acknowledgements}

We thank the participants and their families for their generous donation of information and biospecimens to this study, along with the research staff who recruited them. We acknowledge funding from the National Health and Medical Research Council of Australia (NHMRC, \#APP1026349), along with a number of other philanthropic organisations including Arthritis Australia and the Rebecca Cooper Foundation. The work was supported by the Victorian State Government Operational Infrastructure Program. JAE is supported by an Australian Research Council Future Fellowship. ALP and RS are supported by National Health and Medical Research Council (Australia) Senior Research Fellowships.

\section{Author details}

${ }^{1}$ Genes, Environment \& Complex Disease, Murdoch Childrens Research Institute, 50 Flemington Rd, Parkville, Victoria 3052, Australia. ${ }^{2}$ Department of Paediatrics, The University of Melbourne, Parkville, Vic 3010, Australia. ${ }^{3}$ Arthritis \& Rheumatology, Murdoch Childrens Research Institute, 50 Flemington Rd, Parkville, Victoria 3052, Australia. ${ }^{4}$ Paediatric Rheumatology Unit, Royal Children's Hospital, 50 Flemington Road, Parkville, Victoria 3052, Australia. ${ }^{5}$ Environmental and Genetic Epidemiology Research, Murdoch Childrens Research Institute, 50 Flemington Rd, Parkville, Victoria 3052, Australia. ${ }^{6}$ Paediatric Rheumatology Department, Monash Children's Hospital, Monash Medical Centre, 246 Clayton Road, Clayton, Vic 3168, Australia. ${ }^{7}$ Cancer \& Disease Epigenetics, Murdoch Childrens Research Institute, 50 Flemington Rd, Parkville, Victoria 3052, Australia.

Received: 15 September 2014 Accepted: 11 December 2014 Published: 16 December 2014

\section{References}

1. Ravelli A, Martini A: Juvenile idiopathic arthritis. Lancet 2007, 369:767-778.

2. Herlin MP, Petersen MB, Herlin T: Update on genetic susceptibility and pathogenesis in juvenile idiopathic arthritis. EMJ Rheumatol 2014, 1:73-83.

3. Chistiakov DA, Savost'anov KV, Baranov AA: Genetic background of juvenile idiopathic arthritis. Autoimmunity 2014, 47:351-360.

4. Thompson SD, Marion MC, Sudman M, Ryan M, Tsoras M, Howard TD, Barnes MG, Ramos PS, Thomson W, Hinks A, Haas JP, Prahalad S, Bohnsack JF, Wise CA, Punaro M, Rose CD, Pajewski NM, Spigarelli M, Keddache M, Wagner M, Langefeld CD, Glass DN: Genome-wide association analysis of juvenile idiopathic arthritis identifies a new susceptibility locus at chromosomal region 3q13. Arthritis Rheum 2012, 64:2781-2791.

5. Hinks A, Barton A, Shephard N, Eyre S, Bowes J, Cargill M, Wang E, Ke X, the British Society of Paediatric and Adolescent Rheumatology Study Group,
Kennedy GC, John S, Worthington J, Thomson W: Identification of a novel susceptibility locus for juvenile idiopathic arthritis by genome-wide association analysis. Arthritis Rheum 2009, 60:258-263.

6. Hinks A, Cobb J, Marion MC, Prahalad S, Sudman M, Bowes J, Martin P, Comeau ME, Sajuthi S, Andrews R, Brown M, Chen WM, Concannon P, Deloukas P, Edkins S, Eyre S, Gaffney PM, Guthery SL, Guthridge JM, Hunt SE, James JA, Keddache M, Moser KL, Nigrovic PA, Onengut-Gumuscu S, Onslow ML, Rose CD, Rich SS, Steel KJA, Wakeland EK, et al: Dense genotyping of immune-related disease regions identifies 14 new susceptibility loci for juvenile idiopathic arthritis. Nat Genet 2013, 45:664-669.

7. Cortes A, Brown MA: Promise and pitfalls of the Immunochip. Arthritis Res Ther 2011, 13:101.

8. Ellis JA, Chavez RA, Pezic A, Ponsonby AL, Akikusa JD, Allen RC, Munro JE: Independent replication analysis of genetic loci with previous evidence of association with juvenile idiopathic arthritis. Pediatr Rheumatol Online J 2013, 11:12

9. Ellis JA, Ponsonby AL, Pezic A, Chavez RA, Allen RC, Akikusa JD, Munro JE: CLARITY - ChiLdhood Arthritis Risk factor Identification sTudY. Pediatr Rheumatol Online J 2012, 10:37.

10. Purcell S, Neale B, Todd-Brown K, Thomas L, Ferreira MA, Bender D, Maller J, Sklar P, de Bakker PI, Daly MJ, Sham PC: PLINK: a tool set for wholegenome association and population-based linkage analyses. Am J Hum Genet 2007, 81:559-575.

11. Hinks A, Cobb J, Sudman M, Eyre S, Martin P, Flynn E, Packham J, CAPS, UKRAG, BSPAR, Barton A, Worthington J, Langefeld CD, Glass DN, Thompson SD, Thomson W: Investigation of rheumatoid arthritis susceptibility loci in juvenile idiopathic arthritis confirms high degree of overlap. Ann Rheum Dis 2012, 71:1117-1121.

12. Thompson SD, Sudman M, Ramos PS, Marion MC, Ryan M, Tsoras M, Weiler T, Wagner M, Keddache M, Haas JP, Mueller C, Prahalad S, Bohnsack J, Wise CA, Punaro M, Zhang D, Rose CD, Comeau ME, Divers J, Glass DN, Langefeld CD: The susceptibility loci juvenile idiopathic arthritis shares with other autoimmune diseases extend to PTPN2, COG6, and ANGPT1. Arthritis Rheum 2010, 62:3265-3276.

13. Hinks A, Eyre S, Ke X, Barton A, Martin P, Flynn E, Packham J, Worthington J, CAPS, UKRAG, BSPAR, Thomson W: Association of the AFF3 gene and IL2/IL21 gene region with juvenile idiopathic arthritis. Genes Immun 2010 11:194-198.

14. Reinards THCMAH, Brinkman DMC, Kamphuis SSM, J van Rossum MA, Girschick HJ, Wouters C, Hoppenreijs EPAH, Saurenmann RK, Hinks A, Ellis JA, Bakker E, Verduijn W, Slagboom P, Huizinga TWJ, Toes REM, Houwing-Duistermaat JJ, ten Cate R, Schilham MW: CD226 (DNAM-1) is associated with susceptibility to juvenile idiopathic arthritis. Ann Rheum Dis 2014, (Published Online First 23 July 2014) doi:10.1136/ annrheumdis-2013-205138.

15. Cotsapas C, Voight BF, Rossin E, Lage K, Neale BM, Wallace C, Abecasis GR, Barrett JC, Behrens T, Cho J, De Jager PL, Elder JT, Graham RR, Gregersen P, Klareskog L, Siminovitch KA, van Heel DA, Wijmenga C, Worthington J, Todd JA, Hafler DA, Rich SS, Daly MJ, FOCiS Network of Consortia: Pervasive sharing of genetic effects in autoimmune disease. PLoS Genet 2011, 7:e1002254

\section{doi:10.1186/1546-0096-12-53}

Cite this article as: Chiaroni-Clarke et al:: Independent confirmation of juvenile idiopathic arthritis genetic risk loci previously identified by immunochip array analysis. Pediatric Rheumatology 2014 12:53.

\section{Submit your next manuscript to BioMed Central and take full advantage of:}

- Convenient online submission

- Thorough peer review

- No space constraints or color figure charges

- Immediate publication on acceptance

- Inclusion in PubMed, CAS, Scopus and Google Scholar

- Research which is freely available for redistribution 